\title{
stabilité des cavités de stockage d'hydrocarbures dans le sel
}

\author{
données de l'expérience internationale
}

\author{
par \\ P. Bérest \\ Ingénieur des Mines \\ Laboratoire de Mécanique des Solides, École Polytechnique \\ Division du Sous-sol, Ministère de l'Industrie
}

\section{Facteurs essentiels du comportement des cavités}

Il est sans doute utile, avant d'examiner les leçons qui peuvent être tirées de l'expérience, de recenser les paramètres dont l'influence paraît a priori déterminante :

- le régime des pressions auquel la cavité est soumise : ce paramètre, plus facilement quantifiable, apparaît si important qu'il justifie un paragraphe particulier;

- la forme des cavités: La définition de celle-ci dépend largement des données de la géologie, mais aussi du degré de maîtrise acquis dans le lessivage; on distingue ainsi les cylindres presque parfaits des dômes de sel pur des U.S.A. et des Pays-Bas, les "bouteilles " caractéristiques du lessivage par passes successives de Gaz de France à Tersanne, la forme écrasée de la cavité expérimentale de Kiel, les profils profondément marqués par les blocs d'insolubles ou les hétérogénéités de composition du sel qui sont illustrés par certaines cavités allemandes ou le stockage de Géostock à Manosque (fig. 1);

- la composition des terrains encaissants: En général, le sel est protégé de la dissolution naturelle par d'épaisses couches imperméables et très plastiques (six cents mètres d'argiles à Tersanne); mais la sédimentation du sel est le plus souvent localement interrompue par des bancs rigides d'anhydrite dont les propriétés mécaniques contrastent avec la plasticité du sel. A Manosque, ces couches ont été cassées par les efforts tectoniques, et sont dispersés dans la masse du sel comme des raisins dans un gâteau; à Tersanne les bancs décamétriques, bien corrélés d'un sondage à l'autre, sont restés en place. Dans tous les cas, les blocs désolidarisés par les lessivages sédimentent au fond de la cavité où ils forment une poche constituée de blocs, beaucoup plus rigide que le sel qui en constitue la paroi;
- la distance entre cavités voisines, un site de stockage comprenant en général un grand nombre de cavités (une quarantaine à Manosque, une douzaine à Tersanne et Etrez).

On peut être surpris de voir exclues de cette liste les qualités propres au sel de chaque site. L'analyse microscopique révèle pourtant des différences sensibles de composition et de structure, dont l'influence sur le comportement macroscopique est probable. Mais la quantification des propriétés macroscopiques soulève de graves problèmes de méthode. On réalise de plus en plus clairement que la grande variabilité des résultats est, pour une part, liée aux techniques opératoires ou à des conclusions trop hâtives (articles de J. Bergues et G. Vouille). Les explications fondées sur les différences de comportement des sels gemmes des différents sites apparaissent alors assez arbitraires; c'est la raison pour laquelle nous préférons, sous bénéfice d'inventaire, postuler l'unité du comportement du sel des différents sites.

\section{Stockages de gaz et de liquide}

La stabilité des cavités doit manifestement dépendre de leur profondeur, et de la valeur de leur pression intérieure. L'effet de la profondeur est simple: le sel est un matériel très peu rigide, et l'état de contraintes qui y règne naturellement est très voisin de la pression isotrope qui résulte du poids des terrains surincombants.

Le régime des pressions intérieures dépend essentiellement de la nature des produits stockés. Pour les produits liquides, ou même liquéfiés, le mode d'exploitation ( «balancement par la saumure ») impose la valeur de la pression: celle-ci ne varie que très faiblement autour de la valeur que fixe la colonne de saumure qui remonte jusqu'à la surface et qui permet d'équilibrer les mouvements de produits par des mouvements inverses de saumure. 


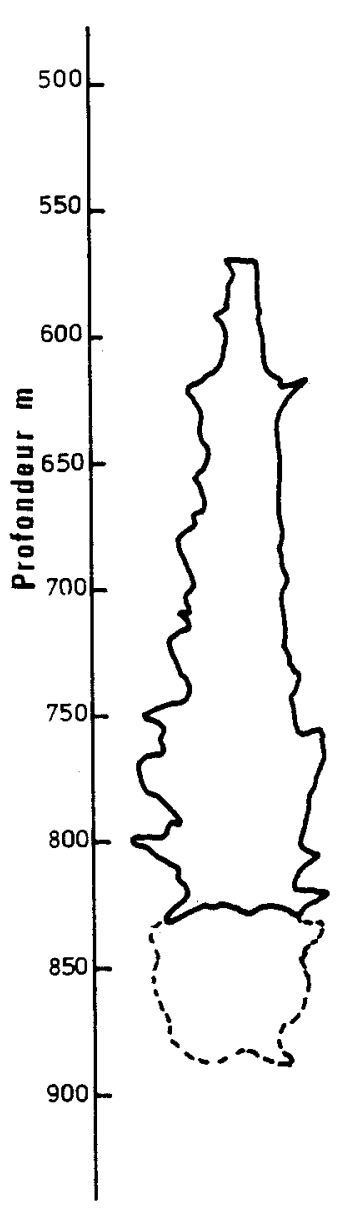

10. MANOSQUE (FRANCE)

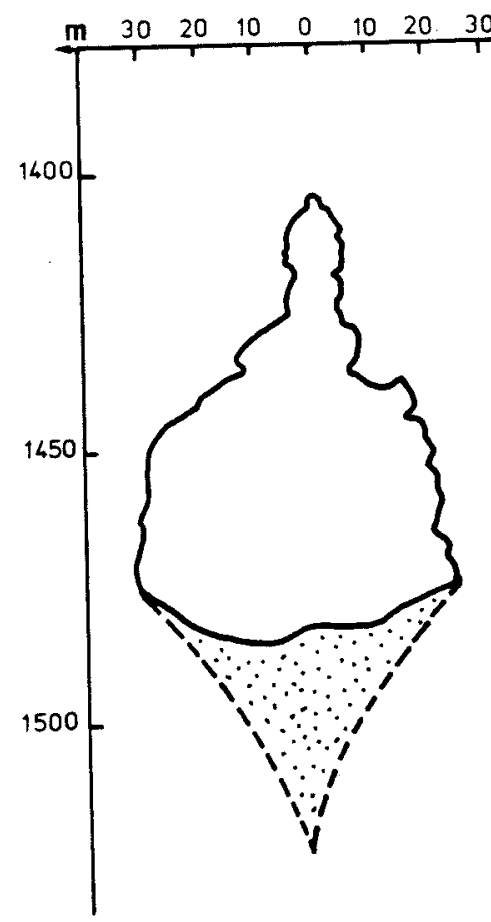

1. TERSANNE (FRANCE)

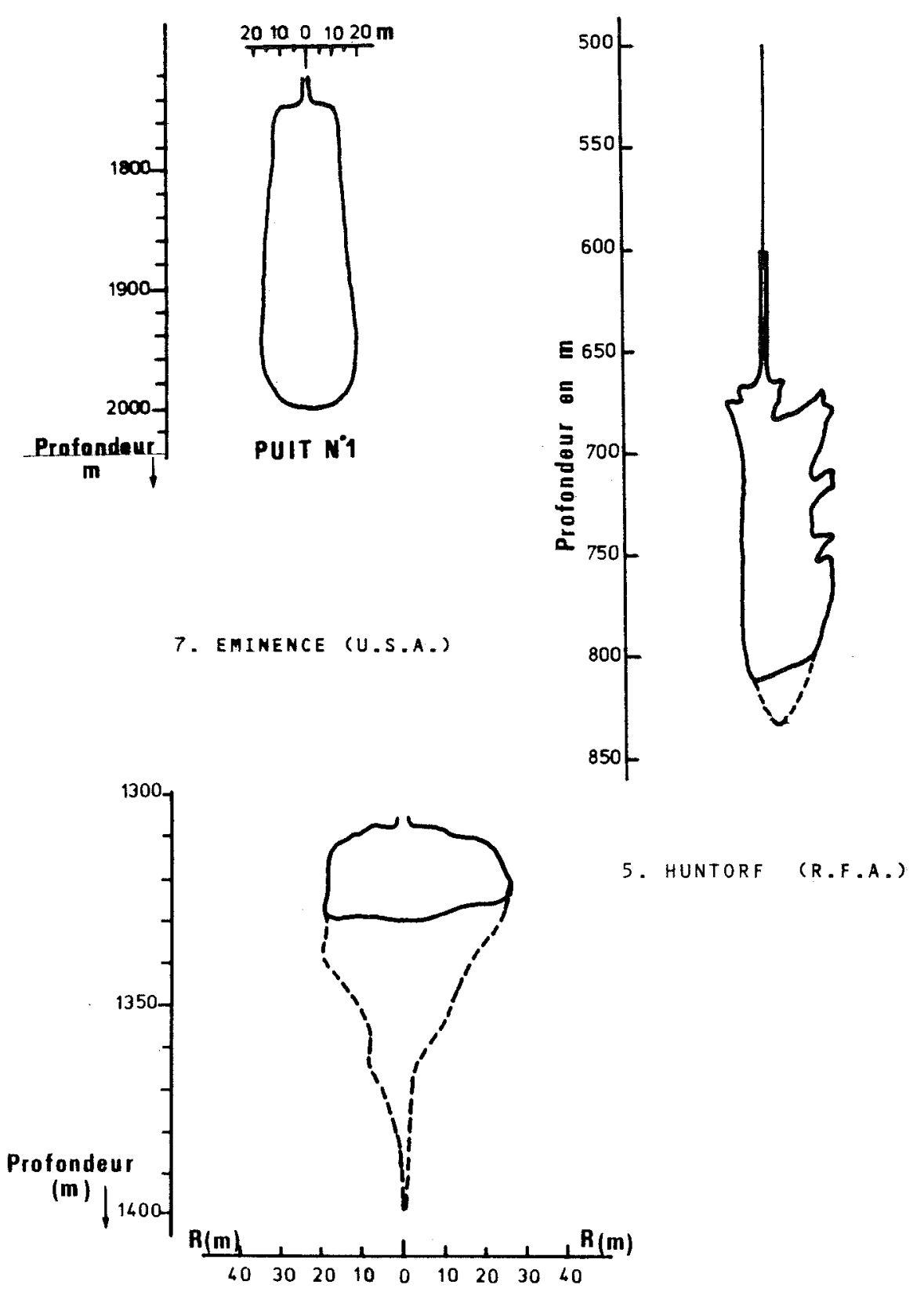

KIEL (R.F.A.)

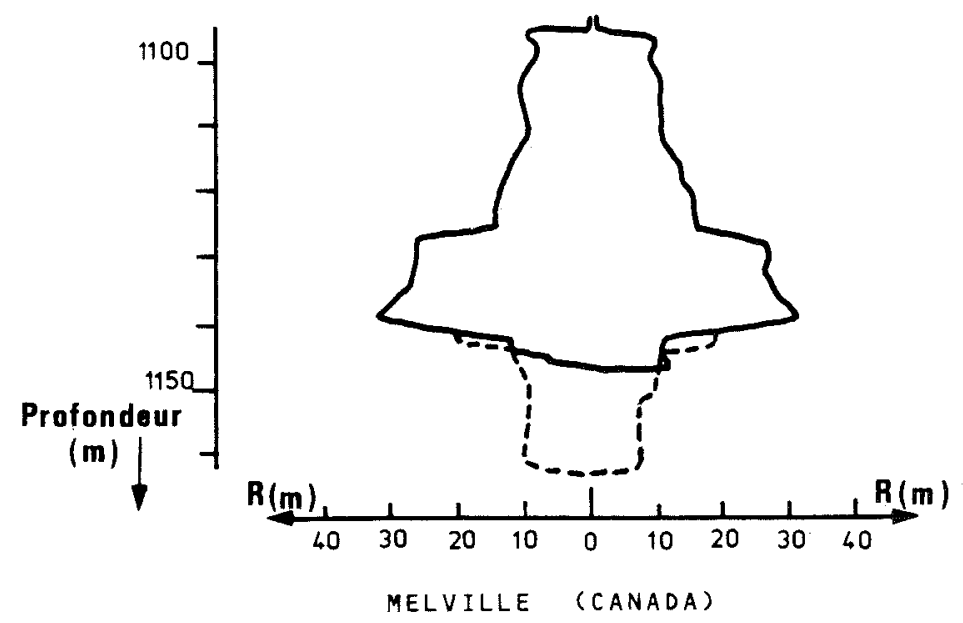

Fig. 1 Formes diverses de cavités 
Dans le cas du gaz naturel, l'élasticité du produit stocké fait dépendre l'intérêt du stockage non seulement du volume des cavités, mais aussi des valeurs extrêmes fixées pour la pression. La pression minimale résulte d'abord de considérations extérieures: elle doit être suffisante pour permettre de déstocker immédiatement sur le réseau sans recompression. Elle doit également assurer un soutènement suffisant des cavités, mais ici les règles sont bien plus délicates à établir. A Tersanne, cette pression minimale $a$ été initialement fixée à $8 \mathrm{MPa}$. La pression maximale est fixée par un souci d'étanchéité de la cimentation du cuvelage: une règle empirique, admise par expérience, limite la pression maximale à une valeur égale au poids d'une colonne de fluide remontant jusqu'à la surface et dont la densité serait égale à 1,66.

II résulte immédiatement de ces règles de fonctionnement que les cavités en gaz sont beaucoup plus fortement sollicitées que les cavités en liquide. En effet :

- la pression minimale y sera beaucoup plus faible : à 1300 mètres de profondeur, elle n'est que la moitié de la pression d'une cavité en liquide;

- le souci d'obtenir une pression maximale suffisante rend plus attractifs les gisements de sel profond: en général, les cavités en gaz seront sensiblement plus profondes que les autres;

- les pressions présentent dans les cavités en gaz des cycles d'amplitude importante (entre $8 \mathrm{MPa}$ et $22 \mathrm{MPa}$ à Tersanne) qui peuvent induire des effets spécifiques analogues à ceux qui résultent de la fatigue des structures métalliques.

Ce dernier aspect étant mis de côté, il est facile de quantifier l'intensité des efforts auxquels sont soumis les cavités. Un critère simple consiste à calculer la différence entre la pression géostatique régnant à la profondeur d'implantation des cavités et la pression intérieure minimale d'exploitation des cavités.

En toute rigueur, ce critère n'a de sens que si le sel possède un seuil de plasticité déterminé par une loi de Von Misès ou de Tresca; mais les expérimentateurs penchent pour des lois de ce type, et ce critère apparaîtra suffisant dans la pratique. La densité des terrains et de la saumure saturée étant respectivement de 2,3 et 1,2, l'indicateur de l'intensité des sollicitations prend la forme suivante:

- pour les stockages de produits liquides:

$$
P_{T}-P_{\text {MIN }}=(0,023-0,012) H=0,011 \mathrm{H}
$$

- pour les stockages de gaz naturel :

$$
P_{T}-P_{\min }=0,023 \mathrm{H}-8
$$

(pressions en $\mathrm{MPa}$, profondeur $\mathrm{H}$ en mètres).

On voit que dès la profondeur de 660 mètres, les cavités en gaz sont plus sollicitées. Les conséquences vont être illustrées par plusieurs exemples.

\section{Données de l'expérience}

Les moyens de mesure du volume des cavités sont limités, indirects; leur précision n'est jamais meilleure que quelques pour cent. La technique qui donne le plus d'information est l'écholog, ou mesure de la forme de la cavité par sonar; mais elle n'est applicable qu'aux cavités pleines de saumure. Pour les cavités en gaz, on peut déterminer le volume global en utilisant l'équation d'état du gaz; les incertitudes cumulées sont importantes et ne peuvent être levées que par des mesures spécifiques (voir l'article de P. Boucly).
Cette incertitude doit être gardée à l'esprit dans le commentaire de la figure 2 . On a représenté 12 cavités implantées dans différents sites dans le monde. Certaines d'entre elles sont de création récente (Etrez, Atwick); on ne dispose pas de renseignements précis sur l'évolution de certaines autres (Epe, Melville, Regina). Les informations recueillies à propos des autres sites peuvent être ainsi résumées:

$1^{\circ}$ pour toutes les cavités existant dans le monde, certaines depuis des dizaines d'années, aucun mouvement de la surface du sol n'a été signalé;

$2^{\circ}$ aucune cavité en liquide ne paraît avoir perdu de volume, à l'intérieur de l'incertitude de $5 \%$ inhérente à la mesure par sonar. Une exception toutefois est constituée par l'exploitation de saumure de Vauvert, au-delà de 2000 mètres de profondeur, dont nous reparlerons;

$3^{\circ}$ les cavités en gaz profondes ont connu des mouvements importants qu'il est utile de détailler :

- Éminence Salt Dome, Mississipi, U.S. A. : La caverne était située entre 1725 mètres et 1965 mètres; le diamètre était de 30 mètres; la pression intérieure variait entre $7 \mathrm{MPa}$ et $28 \mathrm{MPa}$ avec plusieurs cycles annuels.

Après deux ans d'exploitation, le fond était remonté de 36 mètres, la perte totale de volume était de $40 \%$.

- Kiel, R. F. A. : Cette cavité expérimentale était placée entre 1300 mètres et 1400 mètres de profondeur, son volume était de $68000 \mathrm{~m}^{3}$ (dont $28000 \mathrm{~m}^{3}$ d'insolubles foisonnés). On a descendu après lessivage la colonne de saumure par pompage, de sorte que la pression intérieure de la cavité était réduite pratiquement à zéro. Consécutivement à cet essai, le toit s'est effondré sur 3 mètres de hauteur; une mesure de volume effectuée 45 jours après l'essai a montré une perte de volume de $7500 \mathrm{~m}^{3}$; une nouvelle mesure 5 mois plus tard a mis en évidence une perte additionnelle de $1900 \mathrm{~m}^{3}$.

- Tersanne, Drôme, France : Après 10 ans d'exploitation, les cavités $\mathrm{Te} 1$ et $\mathrm{Te} 2$ ont perdu un volume important (voir l'article de P. Boucly). Notons qu'elles étaient situées en 1400 mètres et 1550 mètres et opérées entre $8 \mathrm{MPa}$ et $22 \mathrm{MPa}$.

On peut tirer de ces résultats deux commentaires principaux, qui ont modifié la conception que l'on s'était faite initialement du comportement des cavités dans le sel:

$1^{\circ}$ Les déformations paraissent avoir été progressives et étalées sur plusieurs mois ou années.

$2^{\circ}$ Les pertes de volume ont été beaucoup plus considérables que prévu pour ces 3 groupes de cavité. Pour les autres, le comportement a été globalement conforme aux prévisions. II est remarquable, mais cohérent, que les fortes convergences aient affectées les cavités mécaniquement les plus sollicitées: le paragraphe suivant précisera cette remarque.

\section{Mise en évidence d'un seuil pour le comportement des cavités}

Mettons provisoirement les autres paramètres de côté (forme, amplitude des cycles, etc.) et retenons comme seul critère la différence entre le poids des terrains et la pression intérieure minimale. Les diverses cavités peuvent être de ce point de vue placées dans un diagramme «profondeur-valeur du critère» qui est 


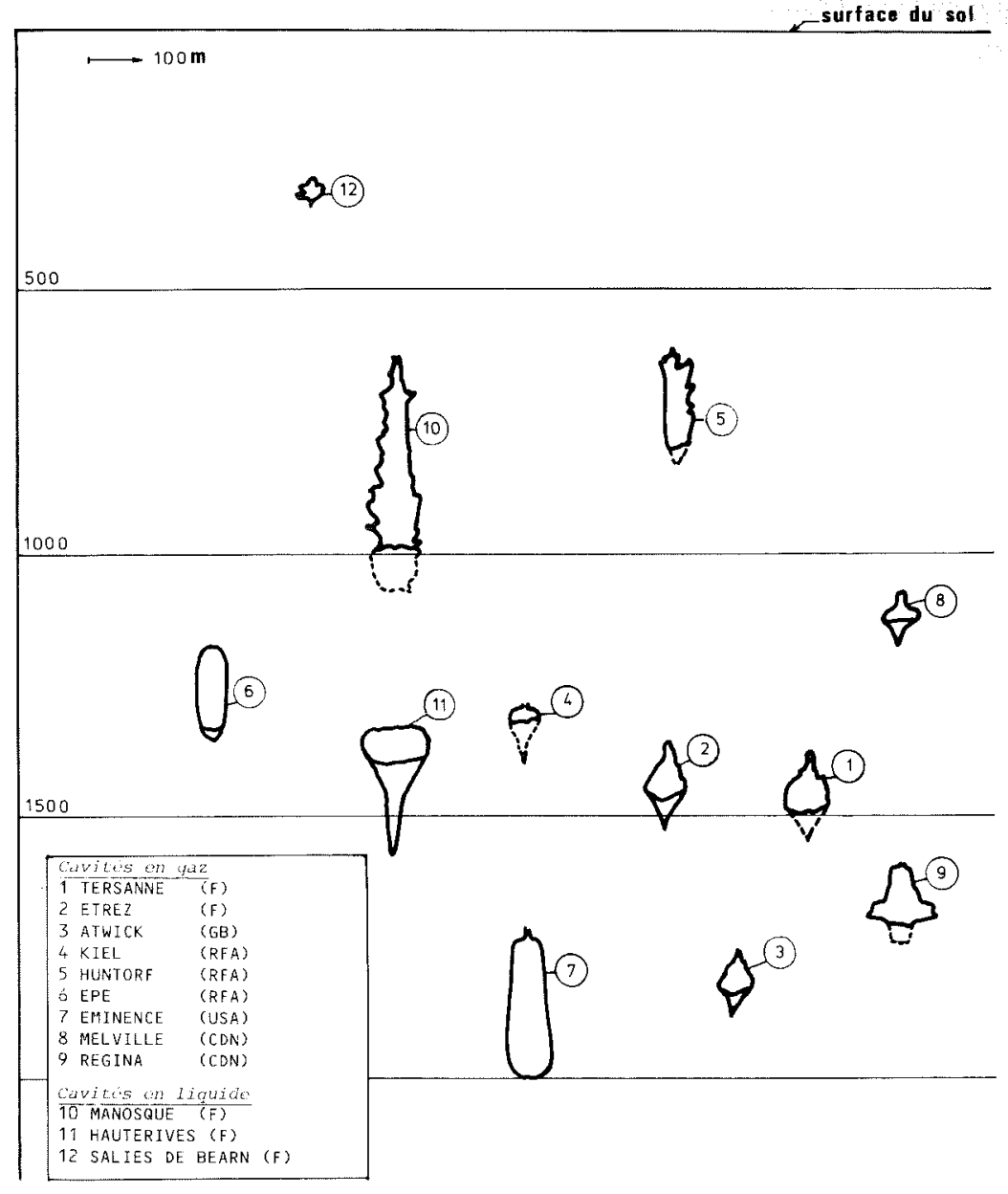

Fig. 2 Exemples de cavités salines dans le monde

donné à la figure 3. Dans ce diagramme, les cavités en liquide sont alignées sur une droite; les cavités en gaz sont représentées par un segment correspondant à la gamme des pressions intérieures utilisées, le point le plus haut figurant la pression intérieure la plus basse.

Une remarque s'impose à l'évidence: les cavités à perte importante de volume sont celles pour lesquelles l'écart entre le poids des terrains et la pression intérieure minimale excède une vingtaine de mégapascal. L'exploitation de saumure de Vauvert, dont nous reparlerons, confirme ce point de vue.

L'aspect différé des phénomènes étant à son tour mis de côté, cette remarque conduit à une conclusion immédiate : la perte de volume étant beaucoup plus que proportionnelle à l'intensité des sollicitations, le comportement des cavités est manifestement de type plastique.

On peut aller plus loin: le modèle le plus simple de comportement plastique est celui d'une sphère creusée dans un massif plastique à critère de Tresca.

Si on note $C$ la cohésion, $E$ le modèle d'élasticité, $v$ le coefficient de Poisson, $\mathrm{P}-\mathrm{P}_{\mathrm{i}}$ l'écart entre poids des terrains et pression intérieure, la perte de volume est caractérisée par l'équation :

$$
\begin{gathered}
Q=\frac{3}{4}\left(P-P_{i}\right) \\
\frac{\Delta V}{V}=\frac{4 C}{E}\left\{(1-2 \wedge) Q+\frac{3}{2}(1-\nu) \exp (Q-1)\right\} .
\end{gathered}
$$

On vérifie que pour les valeurs vraisemblables des paramètres élastiques $\left(v=0,25 \quad E=10^{4} \mathrm{MPa}\right)$ cette formule très simple rend compte de l'allure des phénomènes observés, pourvu que la cohésion retenue soit faible (de l'ordre de $3 \mathrm{MPa}$ ). Cette constatation guide les recherches expérimentales actuelles: lors des premiers essais, la limite de plasticité a sans doute été surestimée, parce que l'influence du temps et aussi sans doute de la température n'ont pas été suffisamment prises en compte (articles de J. Bergues et G. Vouille).

Il est alors logique de compliquer un peu le modèle précédent, en y introduisant une viscosité fonction de la température: on transforme ainsi le modèle de Tresca en modèle de Bingham. L'équation précédente devient alors :

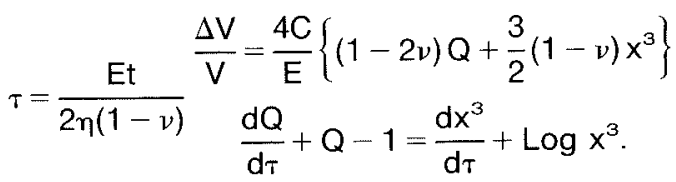

Ce modèle est un cas particulier de celui présenté par N. M. DUC; on retrouve le modèle précédent en faisant $\eta=0$. Si on impose depuis l'instant initial un saut $Q=\left(1+s \frac{4 C}{3}\right) Y(t)$, la solution prend la forme:

$$
\begin{aligned}
& \frac{E t}{2 \eta(1-\nu)}=\int_{\zeta=s}^{\zeta=x^{3}} \frac{d \zeta}{s-\log \zeta} \\
& \frac{1}{3} \frac{\Delta V}{V}=\frac{4 C}{3 E}\left\{(1-2 \nu)(1+s)+\frac{3}{2}(1-\nu) x^{3}\right\} .
\end{aligned}
$$

Dans ce modèle il devient plus difficile d'apprécier la cohésion à partir des résultats de l'expérience, puisqu'on ne sait pas si la totalité de la perte de volume 

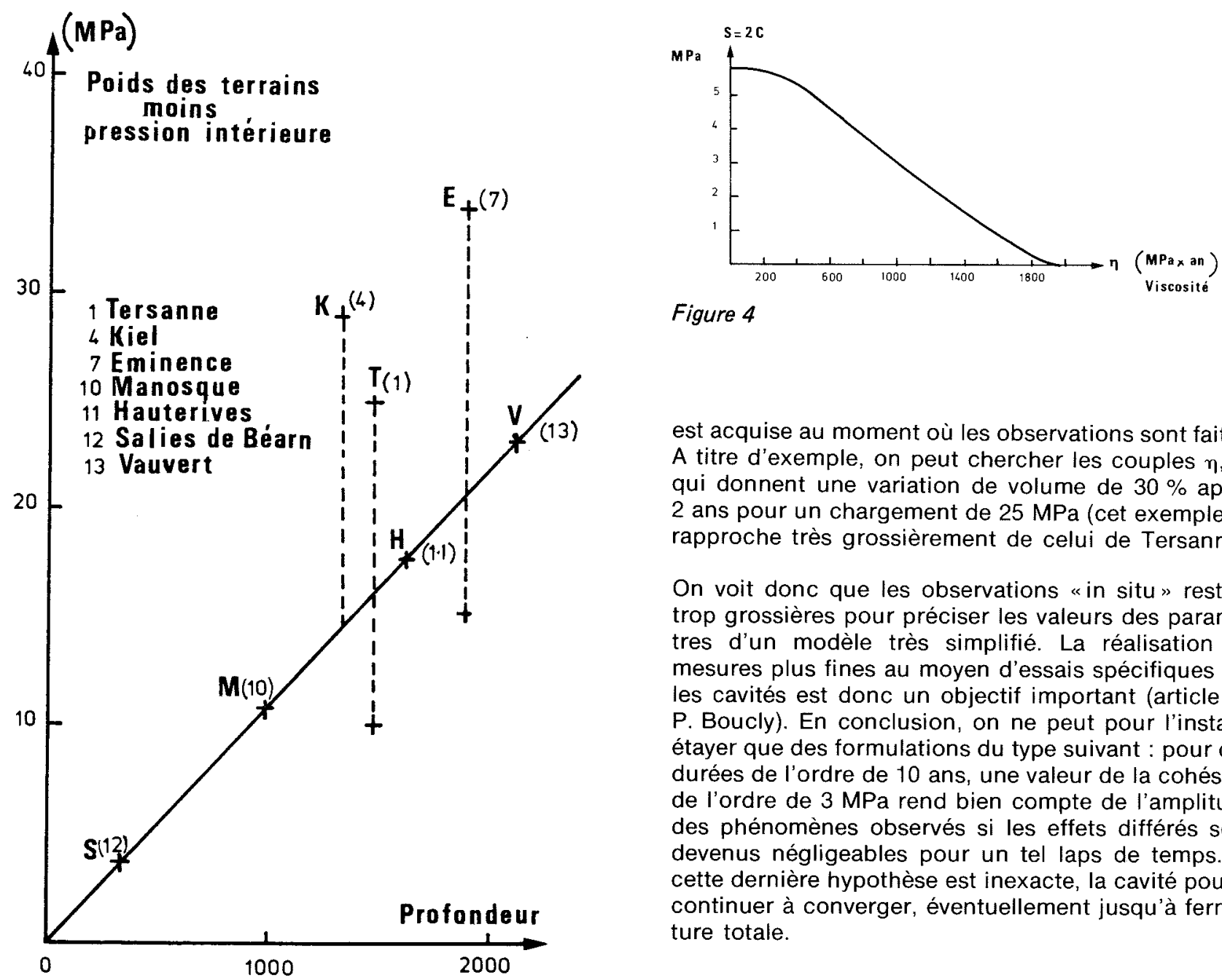

Figure 4

est acquise au moment où les observations sont faites. A titre d'exemple, on peut chercher les couples $\eta, C$, qui donnent une variation de volume de $30 \%$ après 2 ans pour un chargement de $25 \mathrm{MPa}$ (cet exemple se rapproche très grossièrement de celui de Tersanne).

On voit donc que les observations «in situ» restent trop grossières pour préciser les valeurs des paramètres d'un modèle très simplifié. La réalisation de mesures plus fines au moyen d'essais spécifiques sur les cavités est donc un objectif important (article de P. Boucly). En conclusion, on ne peut pour l'instant, étayer que des formulations du type suivant : pour des durées de l'ordre de 10 ans, une valeur de la cohésion de l'ordre de $3 \mathrm{MPa}$ rend bien compte de l'amplitude des phénomènes observés si les effets différés sont devenus négligeables pour un tel laps de temps. Si cette dernière hypothèse est inexacte, la cavité pourra continuer à converger, éventuellement jusqu'à fermeture totale.

Fig. 3 Diagramme profondeur-valeur du critère (différence entre poids des terres et pression intérieure minimale)

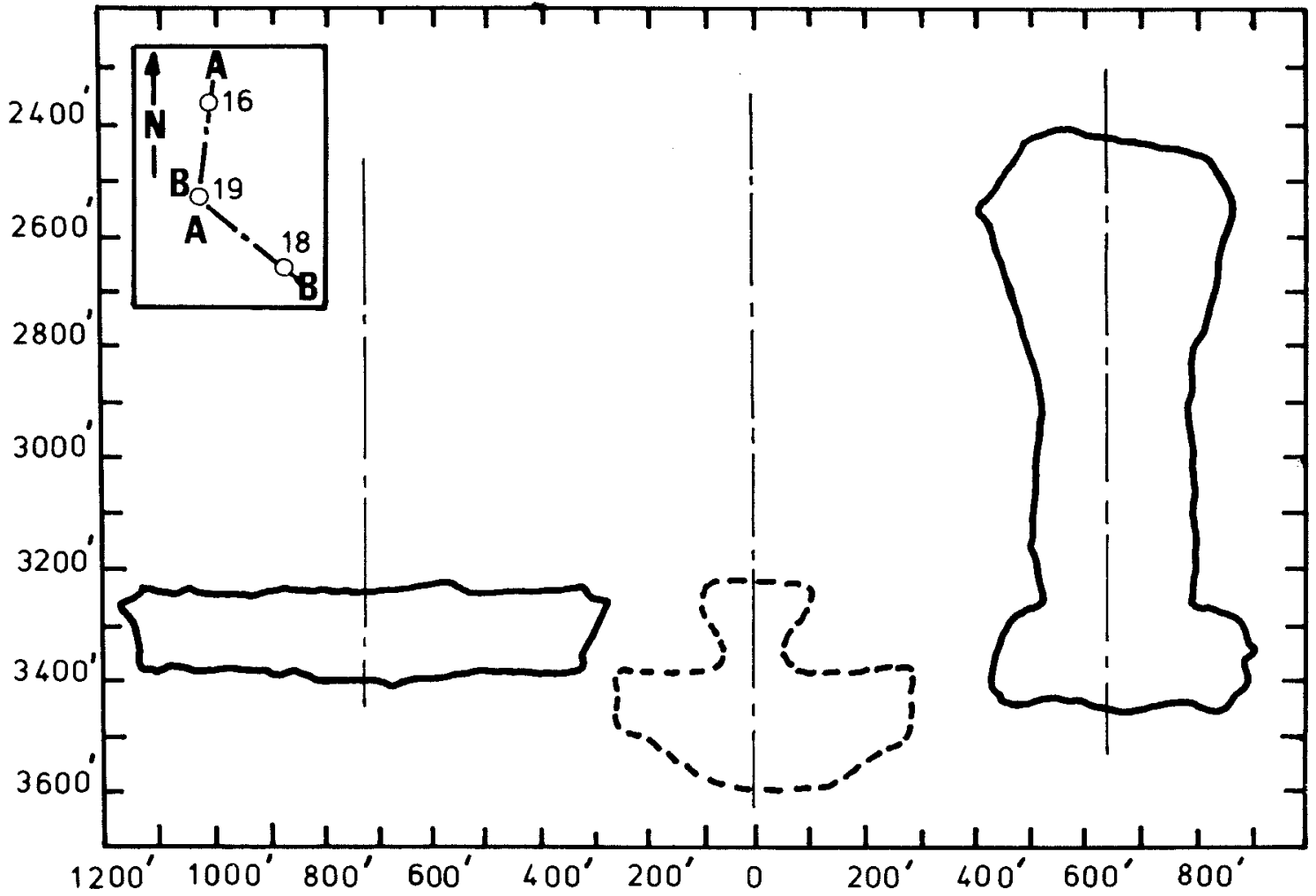

\section{Cavités de west HACKBERRY}

Figure 5 


\section{Le rôle des autres paramètres}

On a donné à "l'écart des pressions » un rôle explicatif de la perte de volume des cavités. D'autres explications ont pu être données, parfois d'apparence un peu farfelue (par exemple, une action spécifique du gaz naturel sur le sel gemme). II reste néanmoins à répertorier ce que peut apprendre l'expérience sur le rôle des autres paramètres.

\subsection{Caractère cyclique du régime des pressions} dans les cavités en gaz

Ce caractère pouvait a priori paraître important (fatigue oligocyclique). Trois faits incitent toutefois à le faire passer au second plan :

- La cavité de Huntorf, profonde de $650 \mathrm{~m}$ à $800 \mathrm{~m}$ fonctionne depuis deux ans en air comprimé avec des cycles journaliers compris entre $5,5 \mathrm{MPa}$ et 7,5 $\mathrm{MPa}$. Aucun phénomène particulier n'y a été observé.

- Le fluage important de Kiel est associé, au contraire de Tersanne et Éminence Salt Dome, à une décharge simple.

- Une comparaison entre le comportement des exploitations de saumure de Vauvert et Hauterives a conduit à vérifier que la première semblait "au-delà du seuil» et la seconde «en-deçà»: le régime des pressions n'y sont pourtant pas cycliques.

Néanmoins, ces présomptions sont encore à accueillir avec prudence : chaque cas cité présente en effet des particularités qui rendent difficiles les comparaisons.

\subsection{Forme des cavités}

Les trois cavités à fort fluage présentent des formes très différentes. On remarque toutefois que le toit plat, de grande portée, de la cavité expérimentale de Kiel doit être défavorable, puisqu'il s'est effondré : rien de semblable n'est arrivé à Tersanne ou Éminence Salt Dome.

Un fait remarquable, encore inexpliqué, doit toutefois être rappelé dans le cas de ces deux dernières cavités: l'essentiel de la perte de volume semble s'être fait par remontée du fond, la forme générale du reste de la cavité n'étant pas modifiée.

\subsection{L'influence des insolubles}

Les bancs épais d'insolubles peuvent jouer un certain rôle. A Manosque, les blocs d'insolubles sont manifestement «expulsés » de la masse salifère et continuent à sédimenter au fond, même longtemps après la fin du lessivage. Les bancs épais de Tersanne renforcent peut-être la symétrie cylindrique du comportement général, les bancs proches du toit semblent avoir bien tenu. En fait, on manque sur ces points de données précises.

\subsection{Distance entre cavités}

Ce point est d'une grande importance pratique et reste très mal élucidé. On a vu que l'expérience conduit à faire admettre une valeur faible de la cohésion du sel. Une conséquence immédiate, que l'on vérifie aisément sur la formule du paragraphe IV, est que la zone plastique doit devenir très étendue; elle dépasse rapidement plusieurs centaines de mètres : sur un site comme celui de Tersanne, il est alors manifeste que les zones plastiques des diverses cavités se rejoignent.
La théorie de la plasticité indique alors que les déformations ne sont alörs plus contenues: l'écoulement libre devient possible. Cette situation devrait être dangereuse, en particulier pour un stockage de gaz, dans lequel les pressions internes des diverses cavités sont indépendantes.

Pourtant l'expérience paraît démentir ce pronostic pessimiste. Les cavités très rapprochées de West Hackberry (U.S.A.) sont utilisées depuis plusieurs années comme stockage stratégique de pétrole (fig. 5) : elles ne semblent pas connaître de phénomènes particuliers. En France, les cavités de l'exploitation de Hauterives sont dans une situation assez semblable, et se comportent normalement. II est donc difficile de conclure: une élémentaire prudence doit conduire toutefois à maintenir des écarts entre axes des cavités suffisants (environ quatre fois le rayon à Tersanne).

\section{Conclusions}

L'examen systématique du comportement des cavités s'enrichira dans les années à venir; les résultats de Nuthermore (R.F.A.) et Atwick (G.-B.) seront particulièrement significatifs en raison de la profondeur de ces cavités. Les principes exposés dans ce présent article conduisent à prévoir qu'elles perdront un volume important.

Si ce pronostic se vérifie, il confortera la première des règles de stabilité qui se dégagent des observations que cet article a résumées :

- l'écart entre le poids des terrains et la pression intérieure minimale ne doit pas excéder de 20 à $22 \mathrm{MPa}$;

- les toits plats de grande portée doivent être évités;

- une distance suffisante (au moins 4 fois le rayon) doit être maintenue entre cavités. 